\title{
A skin-attachable, stretchable integrated system based on liquid GalnSn for wireless human motion monitoring with multi-site sensing capabilities
}

\author{
Yu Ra Jeong ${ }^{1,9}$, Jeonghyun Kim ${ }^{2,3,9}$, Zhaoqian Xie ${ }^{4,5,9}$, Yeguang Xue ${ }^{5}$, Sang Min Won ${ }^{3}$, Geumbee Lee $^{6}$, \\ Sang Woo Jin ${ }^{6}$, Soo Yeong Hong ${ }^{1}$, Xue Feng ${ }^{4}$, Yonggang Huang ${ }^{5,7}$, John A Rogers ${ }^{3,8}$ and Jeong Sook Ha ${ }^{1,6}$ \\ This paper introduces a liquid-metal integrated system that combines soft electronics materials and engineering designs with \\ advanced near-field-communication (NFC) functionality for human motion sensing. All of the active components, that is, strain \\ sensor, antenna and interconnections, in this device are made of liquid metal, and the device has unique gel-like characteristics \\ and stretchability. Patterning procedures based on selective wetting properties of the reduced GalnSn enable a skin-attachable, \\ miniaturized layout, in which the diameter of the device is less than $2 \mathrm{~cm}$. Electromechanical characterization of the strain \\ sensor and antenna reveals their behaviors under large uniaxial tensile and compressive strains, as well as more complex modes \\ of deformation. Demonstrations of these devices involve their use in monitoring various human motions in a purely wireless \\ fashion; examples include wrist flexion, movements of the vocal cord and finger motion. This simple platform has potential for \\ use in human-machine interfaces for prosthetic control and other applications.
}

NPG Asia Materials (2017) 9, e443; doi:10.1038/am.2017.189; published online 27 October 2017

\section{INTRODUCTION}

Skin-mounted, deformable devices capable of sensing various signals such as strain, pressure and temperature can be used in a variety of applications ranging from health monitoring systems and personal diagnostics to human-machine interfaces. ${ }^{1}$ Advanced concepts in stretchable materials and mechanics principles form the basis for devices that can gently laminate onto the soft and curvilinear surfaces of human skin or conformally wrap onto internal organs of the body. ${ }^{2-5}$ Gallium-based liquid metals are highly suitable candidates for such applications due to their unlimited deformability while maintaining excellent metallic conductivity. The use of gallium-based liquid-metal alloys confined in elastomeric enclosures provides intrinsically stretchable properties that maintain bulk electrical conductivity with high stretchability. ${ }^{6}$ Additionally, unlike mercury, gallium is safe to use in ambient environment due to its low vapor pressure. ${ }^{7,8}$ By taking full advantage of the deformability and nontoxicity of the liquid metal, many research groups have utilized liquid metal for wearable applications. Liquid metal has been widely adopted to make a variety of applications such as stretchable microfluidic antenna, ${ }^{9-14}$ soft mechanical sensors, ${ }^{15,16}$ loudspeakers ${ }^{17}$ and ionic current diodes ${ }^{18}$ as well as extensive interconnections. ${ }^{19-22}$

However, a complete liquid-metal system for human-interactive applications, which integrate various functions into one, has yet to be reported. For independent operation of the wearable device, various issues related to signal and power transfer must be solved, and a reliable signal must be generated. In the case of a strain sensor, when wireless transmission functions, including power and data transfer, are not incorporated, a hard wire connection is required with an external measuring device, which degrades the durability of the device and significantly limits the user's activity when worn. Recent work demonstrates various wireless chemical and biological sensor systems with Bluetooth ${ }^{23,24}$ and near-field-communication (NFC) $)^{25-27}$ capabilities, the latter of which can also be operated in a battery-free mode via power harvesting. However, previously reported liquid metal-based

\footnotetext{
${ }^{1}$ Department of Chemical and Biological Engineering, Korea University, Seoul, Republic of Korea; ${ }^{2}$ Department of Electronics Convergence Engineering, Kwangwoon University, Seoul, Republic of Korea; ${ }^{3}$ Department of Materials Science and Engineering, Frederick Seitz Materials Research Laboratory, University of Illinois at Urbana-Champaign, Urbana, IL, USA; ${ }^{4} \mathrm{AML}$, Department of Engineering Mechanics, Center for Mechanics and Materials, Tsinghua University, Beijing, China; ${ }^{5}$ Department of Civil and Environmental Engineering, Mechanical Engineering, and Materials Science and Engineering, Northwestern University, Evanston, IL, USA; ${ }^{6}$ KU-KIST Graduate School of Converging Science and Technology, Korea University, Seoul, Republic of Korea; ${ }^{7}$ Department of Civil and Environmental Engineering, Mechanical Engineering, Materials Science and Engineering, Center for Engineering and Health, and Skin Disease Research Center, Northwestern University, Evanston, IL, USA and ${ }^{8}$ Departments of Materials Science and Engineering, Biomedical Engineering, Chemistry, Mechanical Engineering, Electrical Engineering and Computer Science, and Neurological Surgery; Center for Bio-Integrated Electronics; Simpson Querrey Institute for Nano/biotechnology; Northwestern University, Evanston, IL, USA

${ }^{9}$ These authors contributed equally to this work.

Correspondence: Professor JA Rogers, Northwestern University and Simpson/Querrey Institute, McCormick School of Engineering, Weinberg College of Arts and Sciences, and Feinberg School of Medicine, 2145 Sheridan Road, Evanston, IL 60208, USA.

E-mail: jrogers@northwestern.edu

or Professor JS Ha, Departments of Chemical and biological Engineering, Korea University, 5-1 ka, Anam-dong, Seongbuk-ku, Seoul 136-701, Korea.

E-mail: jeongsha@korea.ac.kr

Received 22 June 2017; revised 8 August 2017; accepted 31 August 2017
} 
antennas tend to be unsuitable for skin-attachable applications in terms of size or thickness, mostly because of limitations in the injection method. Although injecting the liquid metal into microchannels is intuitive and accessible, there are limitations to making delicate design patterns, and additional structure or process is required to integrate liquid-metal channels with other components such as surface mounted devices.

Here, we report a unique, gel-like, liquid-metal system in which all key components-from a strain sensor that can measure tensile and normal strain loading, to an antenna coil that can both receive RF power and transmit data wirelessly, and a collection of electrical interconnections to define the overall circuit topology-consist of lithographically defined conductive traces of liquid phase GaInSn $\left(68.5 \% \mathrm{Ga}, 21.5 \%\right.$ In and $10 \% \mathrm{Sn}$; melting point $\left.=-19^{\circ} \mathrm{C}\right)$, embedded in polydimethylsiloxane (PDMS). The fully wireless, battery-free design, the large operating range (tens of $\mathrm{cm}$ 's) and the thin (sub-millimeter), miniaturized designs (less than $2 \mathrm{~cm}$ in diameter) represent key distinguishing features of the technology, critically enabled by photolithography, chemical reduction and a selective wetting process for patterning features of GaInSn. The system communicates with an external NFC reader that transfers power to the device and receives data from the device in a completely wireless fashion without performance degradation even under large strain (30\%) deformations. The system offers the ability to monitor human motion wirelessly, including wrist flexion and movements of the vocal cords during swallowing and speaking. Simultaneous monitoring of finger motions with three separate strain sensors attached to the joints of individual fingers illustrates multi-site recording of complex motions. The results demonstrate that liquid-metal approaches serve as the basis of integrated, skinmounted wearable platforms with powerful capabilities relevant to clinical medicine, human-machine interfaces and other areas of interest.

\section{MATERIALS AND METHODS}

\section{Fabrication of liquid-metal patterns on PDMS substrate}

A glass substrate was cut into a rectangle $75 \mathrm{~mm} \times 50 \mathrm{~mm}$ in size, followed by rinsing with acetone and isopropyl alcohol. Poly(methyl methacrylate) (PMMA, Microchem, Westborough, MA, USA) was spin-coated (2000 r.p.m., 30 s) and baked at $180^{\circ} \mathrm{C}$ to avoid adhesion between the glass substrate and PDMS. Polydimethylsiloxane (Sylgard 184, Dow Corning Corp., Auburn, MI, USA; mixed at a 10:1 ratio of base to curing agent by weight) was spin-coated on the substrate and partially cured at $75^{\circ} \mathrm{C}$ for $7 \mathrm{~min}$ and $30 \mathrm{~s}$. The $\mathrm{Cr}$ and $\mathrm{Au}$ thin film was deposited on the partially cured PDMS substrates, rather than on the completely cured PDMS, in order to avoid the generation of cracks by thermal residual stress. Deposition of the metal film by sputtering or e-beam evaporation may crack the film surface due to residual strain generated during the film formation process. ${ }^{28,29}$ Therefore, in this study, the $\mathrm{Cr} / \mathrm{Au}$ film layer was deposited on a partially cured PDMS substrate. The optical images of the $\mathrm{PDMS} / \mathrm{Cr} / \mathrm{Au}$ surface are presented in Supplementary Figure S3.

A $10 \mathrm{~nm}$-thick $\mathrm{Cr}$ layer was deposited before depositing the $100 \mathrm{~nm}$-thick Au film $\left(2.1 \AA^{-1}\right)$ using magnetron sputtering (AJA Orion 3 sputter system, MA, USA) on the partially cured PDMS substrates at a rate of $1.0 \AA^{-1}$ to enhance the adhesion between the metal film and PDMS. As-deposited PDMS substrates were placed in an oven at $70^{\circ} \mathrm{C}$ for $20 \mathrm{~min}$ to cure them fully. Solid metal patterns were fabricated using photolithography (AZ 5214 photoresist, spincoating at 3000 r.p.m. for $30 \mathrm{~s}$, baking at $110^{\circ} \mathrm{C}$ for $1 \mathrm{~min} 30 \mathrm{~s}$, UV irradiation for $140 \mathrm{~mJ} \mathrm{~cm}{ }^{-2}$, development for $\approx 28 \mathrm{~s}$ with developer AZ $917 \mathrm{MIF}$ ) and wet etching. A liquid-metal droplet (GaInSn, Rotometals) with a native oxide layer was cast on the Au patterns, followed by casting a few microliters of $10 \mathrm{wt} \%$ $\mathrm{NaOH}$ droplet. The reduced liquid metal was selectively coated on the $\mathrm{Au}$ surface after spreading the reduced GaInSn out on the PDMS substrate. Rinsing and drying were performed using DI water and an oven at $70^{\circ} \mathrm{C}$, respectively.

\section{Fabrication of the liquid-metal NFC device}

A ceramic capacitor (213 pF, Murata Electronics North America, Smyrna, GA, USA), a resistor (2.37 k $\Omega$, Vishay Dale, Shelton, CT, USA), and an NFC chip (SL13A ams AG, USA) were mounted on the liquid-metal pattern using an optical microscope, and electrically connected. PDMS (0.2 g, 10:1 ratio of base to the curing agent by weight) was cast on the substrates and degassed, followed by curing in an oven at $70{ }^{\circ} \mathrm{C}$ for $40 \mathrm{~min}$. a

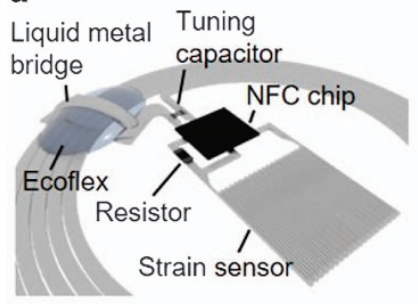

d

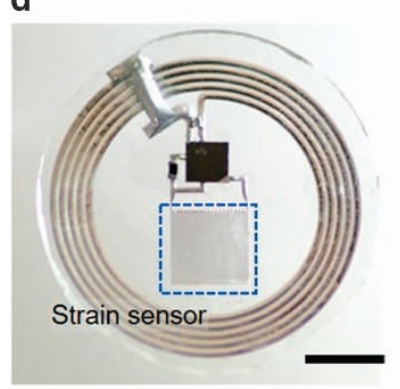

b

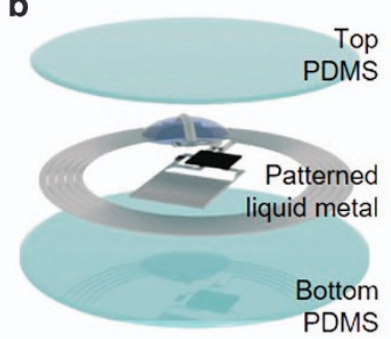

e

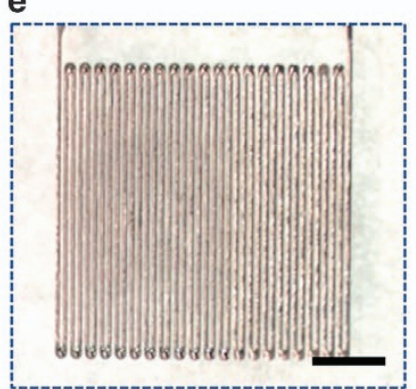

C Wireless power/data transfer via inductive coupling

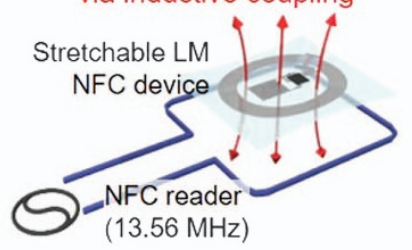

f

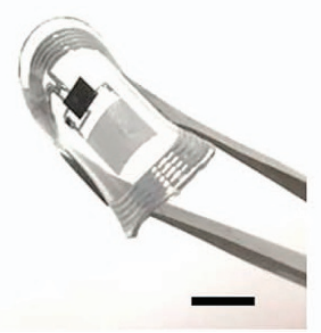

Figure 1 (a) Magnified schematic illustration of the liquid-metal NFC device. (b) Exploded view illustration of the three-layered structure. (c) Illustration of the operating principle. Power is transferred to, and data is transmitted from the liquid-metal (LM) antenna via inductive coupling with a commercial NFC reader. (d) An optical image of the device. Scale bar, $4 \mathrm{~mm}$. (e) A magnified view of the liquid-metal strain sensor. Scale bar, $1 \mathrm{~mm}$. (f) Optical image of the device under deformation with tweezers. Scale bar, $4 \mathrm{~mm}$. NFC, near-field-communication. 


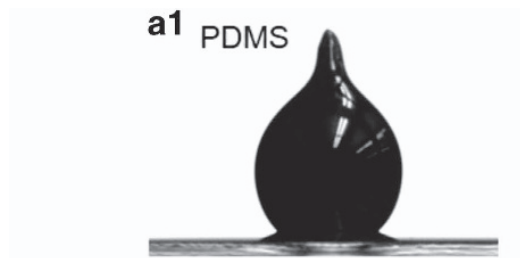

a2
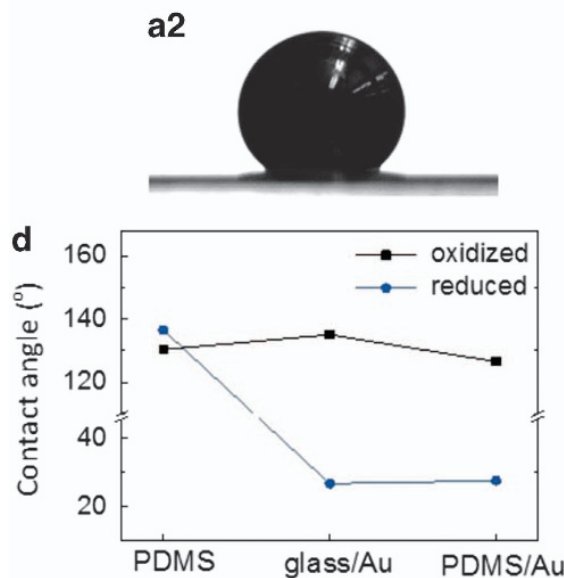

b1

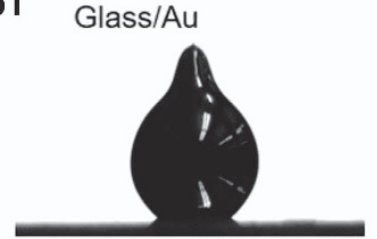

b2
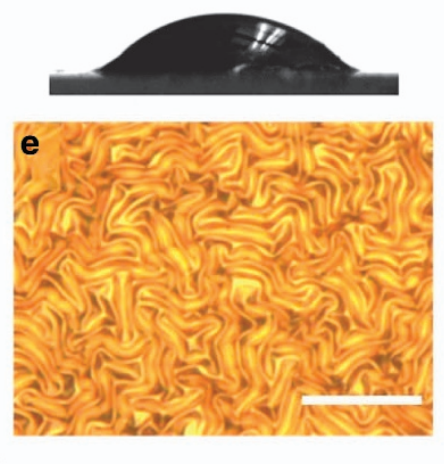

c1

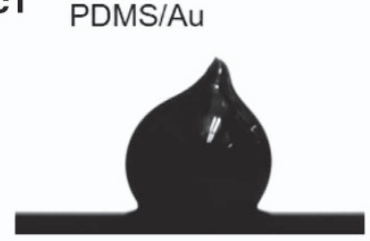

c2
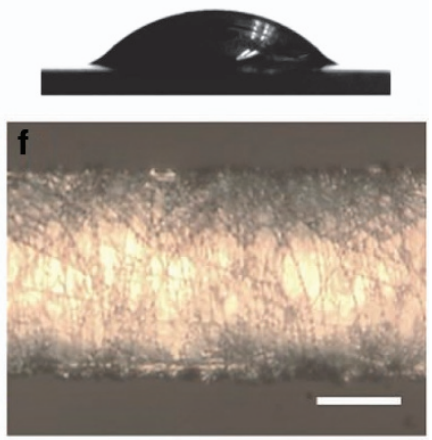

Figure 2 (a-c) Contact angle images of the GalnSn droplet on various substrates of (a) PDMS, (b) Au deposited glass substrate and (c) Au deposited PDMS substrate. Each image shows dramatic changes in contact angle before (al, b1 and $\mathbf{c} 1$ ) and after (a2, b2 and $\mathbf{c} 2)$ reducing its surface with 10 wt\% $\mathrm{NaOH}$. (d) Contact angle measurement of oxidized and reduced GalnSn on various substrates. (e) An optical image of Cr/Au film deposited on partially cured PDMS surface. (f) An optical image of the liquid-metal pattern of $50 \mu \mathrm{m}$ width after encapsulation. Scale bar, $20 \mu \mathrm{m}$. PDMS, Polydimethylsiloxane.

\section{Characterizing the strain sensor}

The electrical resistance was measured with a digital multimeter (DMM, USB-4065, National Instruments, Austin, TX, USA) after inserting a copper wire into the GaInSn microchannel. Mechanical testing of the sensor was performed with a customized uniaxial stretcher. For the dynamic pressure measurements, a force sensor (Mark-10 Series 7) was fixed onto a uniaxial motion controller (SM4-0806-3S), which pressed the sensor in a direction normal to the substrate.

\section{Wireless measurements of various strain}

To monitor various human motion, the devices were mounted on skin after integrating a layer of a thin adhesive (acrylic adhesive, Scapa Healthcare, Inglewood, CA, USA) on the back of the device. The adhesive has biocompatibilities and conformal interfaces with low modulus. ${ }^{26}$ For single device measurement, an NFC reader (AMS Inc., Cupertino, CA, USA) was used for data capture at a distance of $5 \mathrm{~mm}$. For multi-device monitoring, a custombuilt wireless setup was introduced. The communication distance could be extended by applying high power with a large antenna (ID ISC.ANT800/600DA, FEIG Electronics, Duluth, GA, USA) for power transfer and data acquisition. Since the wirelessly obtained data from the custom-built wireless system exhibited code signals with arbitrary units, a calibration graph showing the relationship between two different measurements is shown in Supplementary Figure S19.

\section{Electromagnetic characterization}

Electromagnetic properties were measured with an impedance analyzer (4291A RF impedance/material analyzer, Hewlett Packard, Palo Alto, CA, USA) over a frequency range of 5-20 MHz. Measurements involved placing the device at the center of the primary coil at a vertical distance of $\sim 2 \mathrm{~mm}$.

\section{Measurement of the Young's moduli of the NFC device}

The modulus of the liquid-metal NFC device was measured under uniaxial tensile loading with a dynamic mechanical analyzer (TA Instruments, Q 800, New Castle, DE, USA).

\section{RESULTS AND DISCUSSION}

Figure 1a presents schematic illustrations of a stretchable, wireless strain sensing system based on a liquid-metal configuration: a resistortype strain sensor with an inductive coil that can electromagnetically couple with an external primary coil. All electrical interconnections consist of GaInSn encapsulated within PDMS, thereby forming a three-layer-structure (Figure 1b). Power transfers to the NFC chip from an external NFC reader via inductive coupling and is further delivered to the internal circuits of the device to allow signal acquisition and data transmission from the strain sensor. Since all of the constituent components, except for the commercial surface mounted device chips, have excellent deformability, the device is intrinsically stretchable at the system level, with stable mechanical and electrical properties under external strain. An illustration of the operation appears in Figure 1c, and an optical image is shown in Figure 1d. The coil has five turns of liquid-metal lines with widths and gaps of 460 and $40 \mu \mathrm{m}$, respectively. Detailed information on the circuit design and the coil dimensions are in Supplementary Figure S1. The strain sensor in Figure 1e resides inside the antenna coil and has a width, gap and total length of 70, 40 and $160 \mathrm{~mm}$, respectively. The total thickness, including that of the encapsulating elastomer, is $380 \mu \mathrm{m}$. A cross-sectional scanning electron microscope image of the device revealed by the freeze-fracture method appears in Supplementary Figure S2. An optical image of the device in a state of elastic deformation is shown in Figure 1f.

Figure 2 illustrates the underlying mechanism of patterning liquid metal. The gallium alloy forms a thin oxide layer on its surface in the presence of oxygen at a concentration of over 1 p.p.m. ${ }^{30}$ This oxide shell easily adheres to the surface of almost any material, in contrast to the bare liquid metal, making it difficult to treat and pattern. ${ }^{30,31}$ Various techniques have been developed to pattern liquid metals, including the injection method, stencil lithography, inkjet printing, micro-textured surface enabled patterning and selective wetting. ${ }^{31-35}$ 
a
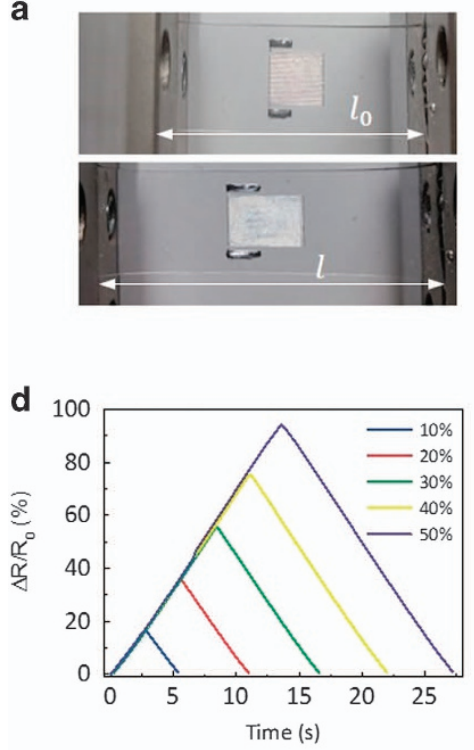

g

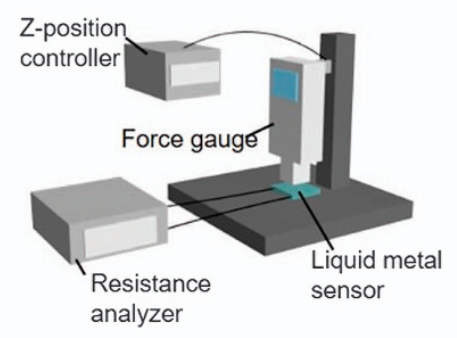

b

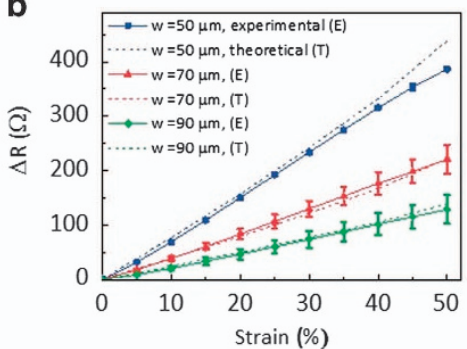

e

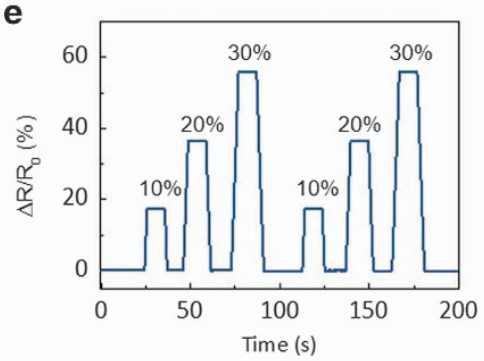

h

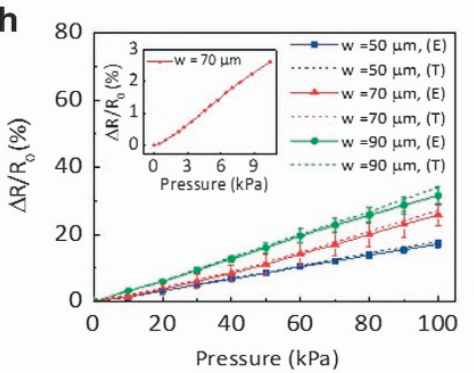

c

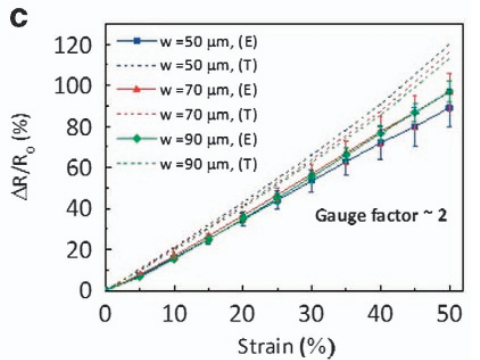

f

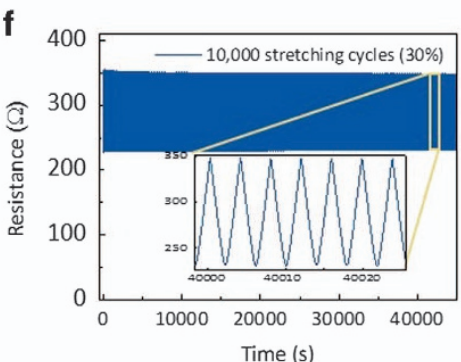

i

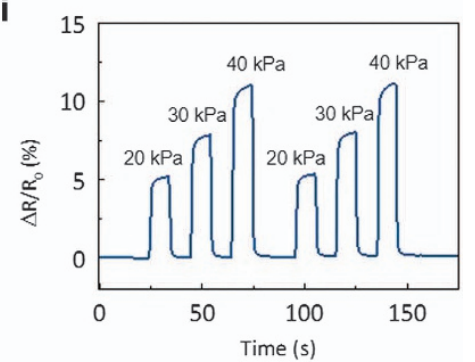

Figure 3 Characterization of the liquid-metal strain gauge. (a) Optical images of the strain sensor before and after stretching by $30 \%$. (b) Measured (solid line) and computed (dotted line) variation of the resistance. $(\Delta \mathrm{R})$ with strain for sensors with various widths. (c) Measured (solid line) and computed (dotted line) variation of the normalized change in the resistance $\left(\Delta R / R_{0}\right)$ with strain. (d) Reversible loading-unloading behavior for various peak strains at a strainloading rate of $0.5 \mathrm{~mm} \mathrm{~s}^{-1}$. (e) Reproducible change in resistance for repeated stretching cycles. (f) Change in resistance as a function of time for repeated strain loading (to 30\%) and unloading for 10000 cycles. The inset shows an enlarged view of the specific time interval highlighted as a yellow rectangular box. (g) Illustration of a setup for the measurements of resistance as a function of normal force (applied pressure). (h) Normalized change in resistance as a function of applied pressure. (i) Reproducible change in resistance for repeated cycles of pressure loading.

Above all, direct injecting liquid metals into the microchannel is the most commonly used and the most reported method. Here, we fabricated patterns of GaInSn liquid-metal electrodes using selective wetting of GaInSn, reduced by exposure to a dilute solution of $\mathrm{NaOH}$, on pre-patterned traces of $\mathrm{Au}$. The oxide layer of the gallium alloys can be reduced by treatment with acid or base, and the bare liquid metal inside the oxide layer can wet the surfaces of certain solid metal materials. $^{35,36}$

Contact angle measurements of a GaInSn droplet before and after treatment with $\mathrm{NaOH}$ on PDMS and an Au-coated surface are shown in Figures $2 \mathrm{a}-\mathrm{d}$. The contact angle was the same on PDMS after treatment with $\mathrm{NaOH}$, whereas it dramatically decreased from $130^{\circ}$ to $27^{\circ}$ on $\mathrm{Au}$ films deposited on glass or PDMS substrates. In the fabrication process, $\mathrm{Cr}$ and Au were deposited on partially cured PDMS substrates to avoid cracking that tends to occur with fully curved substrates. ${ }^{28,29}$ Detailed descriptions of the fabrication process appear in the experimental section in Note S1 (Supplementary Information), as well as in Supplementary Figures S3 and S4. A slightly wavy surface was obtained after the $\mathrm{Cr} / \mathrm{Au}$ deposition process, as shown in Figure 2e, but at a level that was possible to pattern using photolithography and wet etching. This selective wetting approach enables the fabrication of liquid-metal patterns of reduced GaInSn with line widths of $50 \mu \mathrm{m}$, as presented in Figure $2 \mathrm{f}$.
Photolithographic definition of the wetting layers established precise liquid-metal patterns with various designs, outside of the scope of capabilities associated with injection methods due to its need for distinct inlets and outlets. This strategy also enables the facile integration of liquid-metal traces with various components, such as commercial chips without any soldering process, or additional structure, such as a printed circuit board on rigid islands. Supplementary Figure S5 demonstrates the effect of reducing the oxide film of GaInSn on the patterning process, where accurate and immediate patterning can be achieved via treatment of GaInSn with $\mathrm{NaOH}$. The liquid metal immediately wet the gold patterns prepared via the photolithography technique after being reduced by $10 \mathrm{wt} \%$, while the liquid metal without $\mathrm{NaOH}$ treatment did not display selective wetting even after rinsing with isopropyl alcohol. ${ }^{37}$

The electrical properties of the liquid-metal strain sensors, measured under various strains using copper wire connections, are shown in Figure 3. The results include devices with line widths 50, 70 and $90 \mu \mathrm{m}$. The liquid metal embedded in the PDMS deforms linearly with the elastomer under tensile strain loading. Figure 3a presents an optical image of the strain sensor under $30 \%$ uniaxial strain. The resistance can be expressed as a function of the length, width and thickness of the liquid metal. The average resistance values at $0 \%$ strain $\left(R_{0}\right)$ for various widths and lengths are in Supplementary 
Figure S6, where the resistance increases with increasing length and decreasing width. For a fixed volume $(V)$ of the liquid metal, the resistance is $R_{0} \approx l_{0}^{2} /\left(\sigma_{\mathrm{GaInSn}} V\right)$, where $l_{0}\left(=V / A_{0}\right)$ is the wire length for a cross-sectional area $\left(A_{0}\right)$ along the wire and $\sigma_{\mathrm{GaInSn}}$ is the conductivity of the GaInSn $\left(3.46 \times 10^{6} \mathrm{~S} \mathrm{~m}^{-1}\right) .{ }^{38}$ Simulated images of the strain sensors at various levels of tensile strain appear in Supplementary Figure S7.

The sensor exhibits reversible and robust electrical and mechanical performance with minimal hysteresis, thereby enabling reliable operation. The results in Figures $3 \mathrm{~b}$ and $\mathrm{c}$ show that the change in resistance $(\Delta R)$ and the normalized change in resistance $\left(\Delta R / R_{0}\right)$ increase linearly with applied strain $(\varepsilon)$, in good agreement with the theoretical estimate. $\Delta R$ increases with decreasing width, whereas $\Delta R /$ $R_{0}$ is independent of width. Figure $3 \mathrm{~b}$ shows different slopes with varying widths, since each sensor has a different initial resistance at zero strain, $R_{0}$. Figure $3 \mathrm{c}$ was obtained via dividing three curves in Figure $3 \mathrm{~b}$ by each $R_{0}$. The relationship between $\Delta R / R_{0}$ and $\varepsilon$ can be expressed as follows:

$$
\frac{\Delta R}{R_{0}}=\frac{R}{R_{0}}-1=\left(\frac{l}{l_{0}}\right)^{2}-1=\varepsilon^{2}+2 \varepsilon .
$$

The gauge factor is defined by the slope of the normalized resistance curve versus the strain $\varepsilon,\left(\Delta R / R_{0}\right) / \varepsilon$. For $\varepsilon<\sim 30 \%$, the gauge factor is approximately 2 . Strain sensors with three different widths (50, 70 and $90 \mu \mathrm{m}$ ) had the gauge factor of 2 in measurements, with good agreement between the theoretical and experimental results. The sensor shows reversible behavior for various strain loading and unloading cycles, with negligible hysteresis, as supported by the time-resistance data collected at a loading speed of $0.5 \mathrm{~mm} \mathrm{~s}^{-1}$ in Figures $3 \mathrm{~d}$ and e. The sensor also has excellent durability, showing stable electrical performance for 10000 stretching cycles under $30 \%$ strain, as presented in Figure $3 \mathrm{f}$. The inset of Figure $3 \mathrm{f}$ is an enlarged view of the specific time interval, which exhibits reliable performance on repetitive stretching. The sensitivity to temperature is insignificant (Supplementary Figure S8), with only 5\% increase in resistance for an increase in temperature from 20 to $50^{\circ} \mathrm{C}$, and a gauge factor that remains unchanged. The resistance decreases slightly upon stretching in a direction perpendicular to the sensor lines, showing a $5.2 \%$ decrease under 50\% strain (Supplementary Figure S9). Such behavior likely arises from reductions in length $(l)$ due to the Poisson effect.

The sensor can also detect loading associated with applied pressure. A schematic illustration of the measurement setup for characterizing this response is presented in Figure 3g. A commercial force gauge (Mark-10) fixed on a Z-stage controller with a $3.3 \times 3.3 \mathrm{~mm}^{2}$ tip applied to the end served as a controlled source of pressure. Detailed descriptions of the measurement setup, including the support platform and the force actuator, are described in Note S5 (Supplementary Information) and Supplementary Figure S10. For pressures up to $100 \mathrm{kPa}$, the resistance shows a linear increase, where the slope of $\Delta R /$ $R_{0}$ versus pressure increases with increasing line width (Figure $3 \mathrm{~h}$ ). Changes in the resistance over a pressure loading-unloading cycle are
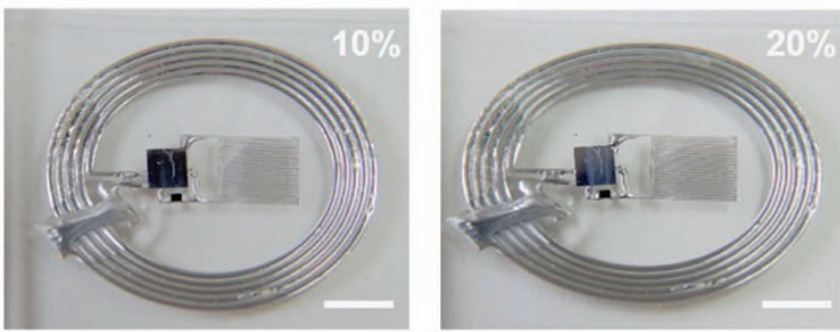

b
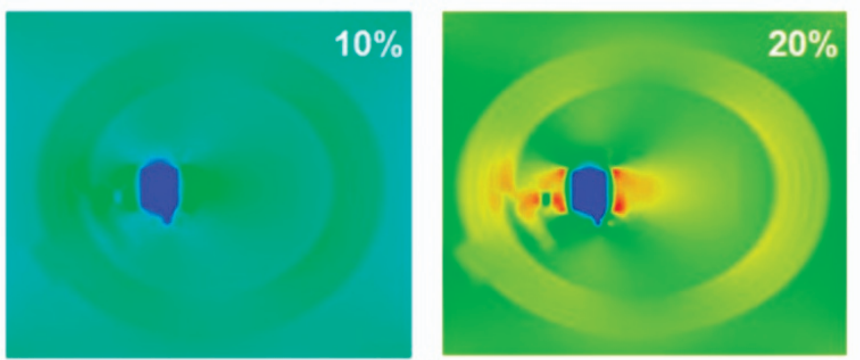
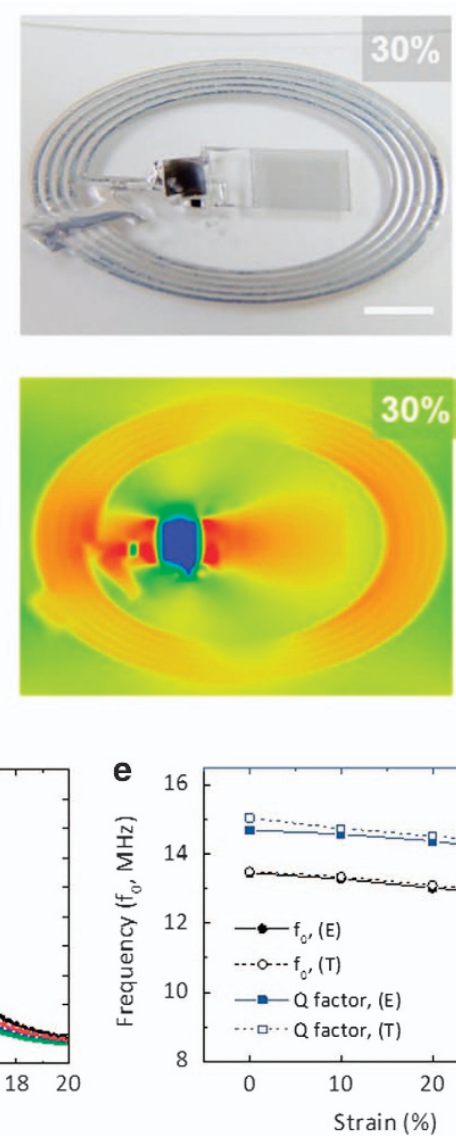

Strain(\%)

32

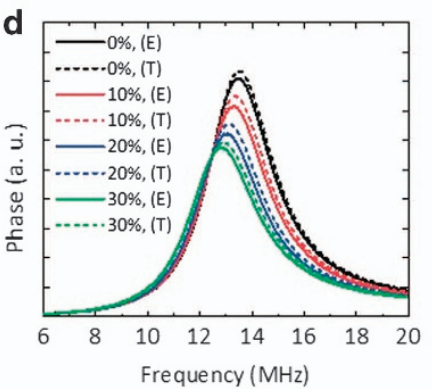

Figure 4 Mechanical and electromagnetic properties of the stretchable liquid-metal antenna. (a) Optical images of the device under uniaxial strain up to $30 \%$. Scale bar, $4 \mathrm{~mm}$. (b) FEA results for the stress distributions associated with devices under uniaxial stretching up to $30 \%$. (c) Change in the resistance of the NFC inductive coil under uniaxial strain. (d) Phase response under uniaxial strain. (e) Change in the resonance frequency and $Q$ factor under uniaxial strain. Experimentally measured and theoretically calculated data are shown with solid and dotted lines, respectively. FEA, finite element analysis. 
a

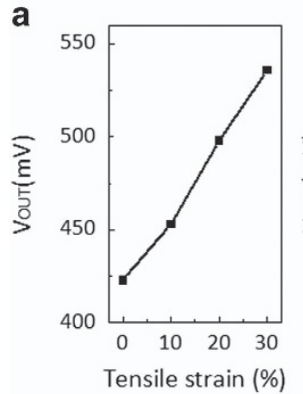

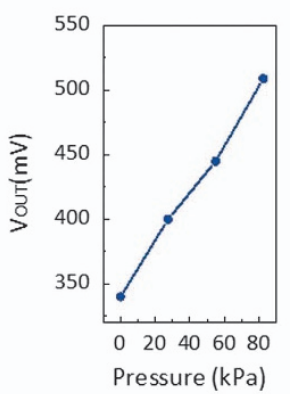

b

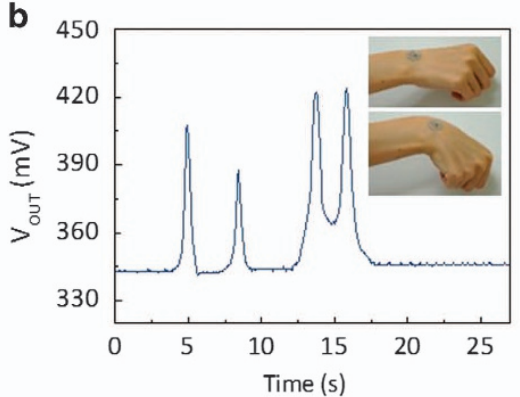

C

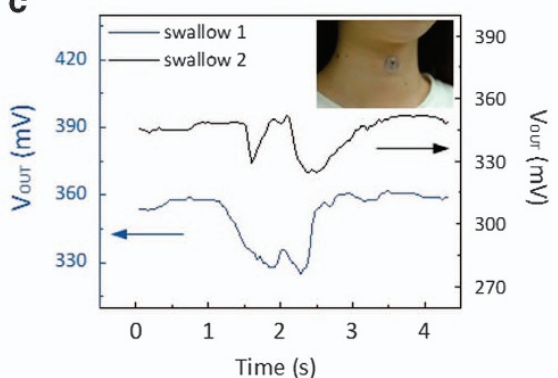

$\mathbf{f}$

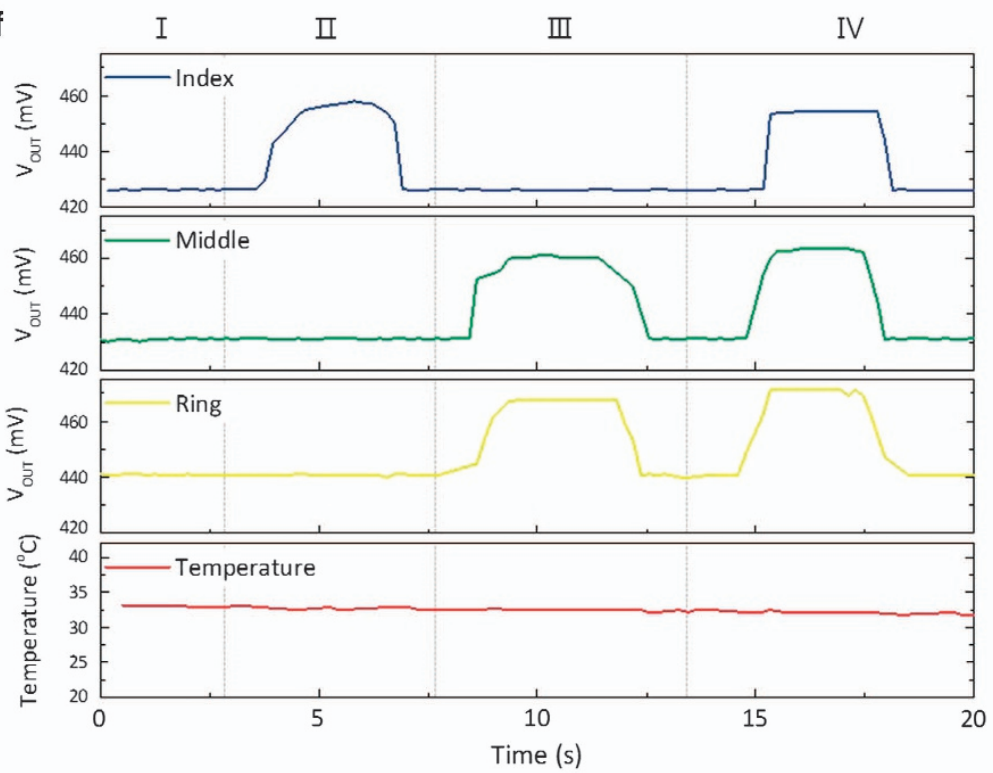

d

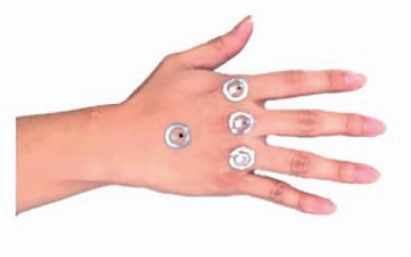

e

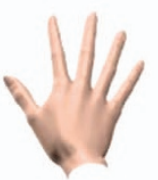

I

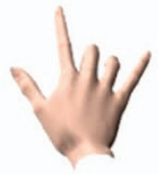

III

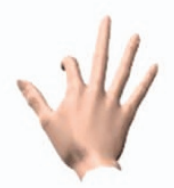

ㅍ

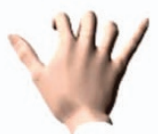

IV

Time (s)

Figure 5 Wireless monitoring of external strain loading and various human motions. (a) Wireless measurement of the output voltage under uniaxial tensile strain and pressure loading. The device was stretched up to $30 \%$ and pressed up to $80 \mathrm{kPa}$. (b) Wireless detection of wrist flexion, with corresponding inset optical image of the wrist motion. (c) Real-time wireless monitoring of muscle motion upon swallowing. The inset shows an image of a device attached to the throat. (d) Optical images of the devices mounted on the joints of each finger and on back of the hand. Scale bar, $5 \mathrm{~cm}$. (e) Schematic illustration of four different finger motions. (f) Motion detection of the index, middle and ring fingers with a simultaneous measurement of skin temperature.

reproducible for various pressure values, and the resistance returns its original value when the sensor is unloaded to $0 \mathrm{~Pa}$ (Figure 3i). The magnitudes of the changes in resistance depend on the Young's modulus of the encapsulating polymer. The experimental and theoretical results in Supplementary Figure S11 both show that the resistance changes are greater when the sensor is encapsulated within a lower modulus silicone material (Ecoflex, Young's modulus $\sim 125 \mathrm{kPa}$ ). Some hysteresis is observed upon pressure loading and unloading, whereas negligible hysteresis occurs in the case of stretching (Supplementary Figure S12). Stress-strain curves of the sensor and bare PDMS, shown in Supplementary Figure S13, do not exhibit any hysteresis, indicating that the viscoelasticity of the PDMS elastomer is not a major factor in the electrical hysteresis. Such different hysteresis behavior between the tensile and compressive strains could be attributed to the convex geometry of the microchannel, where the flow-back of the liquid metal into the channel may be limited due to its viscosity. ${ }^{16,39}$

The use of a GaInSn antenna for power transmission and data communication also provides stable electrical and mechanical performance under stretching and other modes of deformation. Figures $4 \mathrm{a}$ and $\mathrm{b}$ show optical images of the antenna under uniaxial tension up to $30 \%$ stretching, and the strain distribution obtained from the finite element analysis (see Note S2 in Supplementary Information), respectively. The maximum strain of $32 \%$ occurs around the NFC chip $(2.38 \mathrm{~mm} \times 2.38 \mathrm{~mm}$; thickness of $\sim 100 \mu \mathrm{m})$ when the applied strain reaches $30 \%$. The Young's modulus of the device is $3.8 \mathrm{MPa}$, calculated by the strain-stress curve of the device presented in Supplementary Figure S14. The resistance of the inductive coil increases with stretching, as shown in Figure $4 \mathrm{c}$ and Supplementary Figure S15. The phase response, resonance frequency and Q factor have some minor dependence on strain (Figures $4 \mathrm{~d}$ and e). In particular, the resonance frequency and $\mathrm{Q}$ factor decrease by $5 \%$ (from 13.5 to $12.8 \mathrm{MHz}$ ) and $10 \%$ (from 4.3 to 3.9 ), respectively, for stretching up to $30 \%$. All measurements are in good agreement with simulation. These changes in performance are caused by the deformation of the shape in the coil antenna, consistent with behaviors in circular copper antenna structures. ${ }^{25,40}$ However, these characteristics do not have significant adverse effects on the operation of the wireless strain sensor using NFC, since the integrated NFC chip has a constant output voltage of $3.4 \mathrm{~V}$. Only signals related to the resistance change $(\Delta R)$ of the sensor caused by the external strain loading are transmitted to the external reader as meaningful data.

The overall system can wirelessly communicate with an external reader via magnetic inductive coupling at a frequency of $13.56 \mathrm{MHz}$. The device is designed to resonate near $13.56 \mathrm{MHz}$ by proper selection of a tuning capacitor $\left(C_{t}\right)$ connected in an electrically parallel fashion 
with the inductive coil. The wirelessly received AC power from the antenna coil is rectified through the NFC chip, and a constant voltage $\left(V_{\text {EXT }}\right)$ is applied to a serially connected circuit that consists of a commercial resistor and the liquid-metal strain sensor, as shown in the circuit diagram in Supplementary Figure S1a. The voltage applied to the strain sensor $\left(V_{\text {OUT }}\right)$ is determined by the ratio between the resistance of the commercial resistor $(R 1)$ and that of the liquid-metal strain sensor. The signal is continuously transmitted to the NFC chip, and eventually transmitted to an external reader at a sampling rate of $25 \mathrm{~Hz}$.

The wirelessly measured output voltages $\left(V_{\mathrm{OUT}}\right)$ corresponding to applied tensile and compressive strain are presented in Figure 5a. To demonstrate wireless function, we attached the device onto human skin and obtained real-time data from the strain sensor induced by deformations of the device, each corresponding to a specific motion. For the device mounted on skin (thickness $1 \mathrm{~mm}$ ) subjected to uniaxial tension and to approximately $10 \%$ stretching, the maximum normal and shear stresses at the device/skin interface were below the threshold for somatosensory perception of forces $(20 \mathrm{kPa}),{ }^{41}$ as shown in Supplementary Figure S16. Figure 5b shows detection associated with wrist flexion and release for a device mounted on the wrist. The movements of the vocal cords can be monitored by attaching the device on the throat. Figure $5 \mathrm{c}$ shows the change in the output voltage ( $V_{\text {OUT }}$ ) while swallowing, where unique waveforms with two concave peaks correspond to two different measurements. Similar responses can be recorded using wired connections, as shown in Supplementary Figure S17. Similarly, the movements of the vocal cords and muscle motion in speech can also be monitored when speaking specific words, thereby yielding distinguishable waveforms (Supplementary Figure S18).

To demonstrate scalability in sensing functionality, three devices mounted on the joints (carpometacarpal joints) of the index, middle and ring fingers provide data simultaneously while moving the fingers. An additional device attached to the back of the hand can monitor the temperature of the skin wirelessly using the internal temperature sensor in the NFC chip. For multi-device monitoring, we introduced a custom-built system that can communicate with multiple NFC devices at the same time, and increase communication distance up to approximately $30 \mathrm{~cm}$ by use of a high power RF system $(8 \mathrm{~W})$. The details are in the experimental procedure in Note S1 (Supplementary Information). Optical images of the hand and schematic illustrations of each motion are presented in Figures $5 \mathrm{~d}$ and e. Signals from four different motions as well as the temperature of the skin can be obtained via the NFC interface. Each motion is clearly distinguishable in real-time data, as shown in Figure 5f. The data received by the custom-built system were calibrated into voltage units $(\mathrm{mV})$, as shown in Figure 5f, since it receives signals with hexadecimal codes with arbitrary units in contrast to the commercial reader $\left(V_{\text {OUT }}, \mathrm{mV}\right)$. A fitted curve showing the relationship between two different measurements is presented in Supplementary Figure S19. For an improved understanding of the finger motions, wired measurements of the finger motions identical those in Figure $5 \mathrm{f}$ are presented in Supplementary Figure S20. Additionally, the quantitative values for the strain associated with the degree of flexion and the corresponding response of the resistance are shown in Note S4 (Supplementary Information) and Supplementary Figure S21.

\section{CONCLUSION}

For the first time, we demonstrate a skin-attachable, gel-like stretchable integrated system based on liquid GaInSn for wireless human motion monitoring with multi-site sensing capabilities. The devices include signal acquisition and data transmission functions as well as strain sensors and interconnections, all composed of liquid metal. Liquid-metal patterns were obtained by using photolithography and selective wetting properties of the reduced GaInSn as a precise means for integration, with key advantages compared to other fabrication methods. Examples of motion monitored using this system include wrist flexion and motion of the vocal chords during swallowing and speech. Moreover, various finger motions can be distinguished by mounting multiple devices on the finger joints. The combination of liquid-metal-enabled deformability and the untethered sensing of dynamic motion achieved via NFC technology creates many possibilities in wearable devices, with high potential as a human-robotic interface for prosthetics.

\section{CONFLICT OF INTEREST}

The authors declare no conflict of interest.

\section{ACKNOWLEDGEMENTS}

This work was supported by the National Research Foundation of Korea (NRF) grant funded by the Korean government (MEST) (Grant No.

NRF-2016R1A2A1A05004935). We also thank the KU-KIST graduate school program of Korea University.

Author contributions: YRJ, JK and ZX contributed equally to this work. Conceptualization, YRJ, JK, JSH and JAR; Methodology, YRJ, JK, SMW, GL, SWJ, SYH; Formal analysis, YRJ, ZX, YX, XF, YH; Investigation, YRJ, JK; All the authors contributed to interpretation of the results and writing of the manuscript. JAR and JSH supervised the work.

\section{PUBLISHER'S NOTE}

Springer Nature remains neutral with regard to jurisdictional claims in published maps and institutional affiliations.

1 Lochner, C. M., Khan, Y., Pierre, A. \& Arias, A. C. All-organic optoelectronic sensor for pulse oximetry. Nat. Commun. 5, 5745 (2014).

2 Webb, R. C., Bonifas, A. P., Behnaz, A., Zhang, Y., Yu, K. J., Cheng, H., Shi, M., Bian, Z., Liu, Z., Kim, Y.-S., Yeo, W.-H., Park, J. S., Song, J., Li, Y., Huang, Y. Gorbach, A. M. \& Rogers, J. A. Ultrathin conformal devices for precise and continuous thermal characterization of human skin. Nat. Mater. 12, 938-944 (2013).

3 Chou, H.-H., Nguyen, A., Chortos, A., To, J. W. F., Lu, C., Mei, J., Kurosawa, T. Bae, W.-G., Tok, J. B. H. \& Bao, Z. A chameleon-inspired stretchable electronic skin with interactive colour changing controlled by tactile sensing. Nat. Commun. 6, 8011 (2015).

4 Kaltenbrunner, M., Sekitani, T., Reeder, J., Yokota, T., Kuribara, K., Tokuhara, T. Drack, M., Schwodiauer, R., Graz, I., Bauer-Gogonea, S., Bauer, S. \& Someya, T. An ultra-lightweight design for imperceptible plastic electronics. Nature 499, 458-463 (2013).

5 Yamada, T., Hayamizu, Y., Yamamoto, Y., Yomogida, Y., Izadi-Najafabadi, A., Futaba, D. N. \& Hata, K. A stretchable carbon nanotube strain sensor for humanmotion detection. Nat. Nanotechnol. 6, 296-301 (2011).

6 Dickey, M. D. Stretchable and soft electronics using liquid metals. Adv. Mater. 29, 1606425 (2017).

7 Dickey, M. D., Chiechi, R. C., Larsen, R. J., Weiss, E. A., Weitz, D. A. \& Whitesides, G. M. Eutectic Gallium-Indium (EGaln): a liquid metal alloy for the formation of stable structures in microchannels at room temperature. Adv. Func. Mater. 18, 1097-1104 (2008).

8 Cochran, C. N. \& Foster, L. M. Vapor pressure of gallium, stability of gallium suboxide vapor, and equilibria of some reactions producing gallium suboxide vapor. J. Electrochem. Soc. 109, 144-148 (1962).

9 Entesari, K. \& Saghati, A. P. Fluidics in microwave components. IEEE Microwave Mag. 17, 50-75 (2016).

10 Qusba, A., RamRakhyani, A. K., So, J. H., Hayes, G. J., Dickey, M. D. \& Lazzi, G. On the design of microfluidic implant coil for flexible telemetry system. IEEE Sensors J. 14, 1074-1080 (2014).

11 Kubo, M., Li, X., Kim, C., Hashimoto, M., Wiley, B. J., Ham, D. \& Whitesides, G. M. Stretchable microfluidic radiofrequency antennas. Adv. Mater. 22, 2749-2752 (2010).

12 So, J.-H., Thelen, J., Qusba, A., Hayes, G. J., Lazzi, G. \& Dickey, M. D. Reversibly deformable and mechanically tunable fluidic antennas. Adv. Func. Mater. 19, 3632-3637 (2009).

13 Jeong, S. H., Hjort, K. \& Wu, Z. Tape transfer atomization patterning of liquid alloys for microfluidic stretchable wireless power transfer. Sci. Rep. 5, 8419 (2015). 
30 Doudrick, K., Liu, S., Mutunga, E. M., Klein, K. L., Damle, V., Varanasi, K. K. \& Rykaczewski, K. Different shades of oxide: from nanoscale wetting mechanisms to contact printing of gallium-based liquid metals. Langmuir 30, 6867-6877 (2014).

14 Cheng, S. \& Wu, Z. Microfluidic stretchable RF electronics. Lab Chip 10, 3227-3234 (2010).

15 Matsuzaki, R. \& Tabayashi, K. Highly stretchable, global, and distributed local strain sensing line using GalnSn electrodes for wearable electronics. Adv. Func. Mater. 25, 3806-3813 (2015)

16 Park, Y. L., Chen, B. R. \& Wood, R. J. Design and fabrication of soft artificial skin using embedded microchannels and liquid conductors. IEEE Sensors J. 12, 2711-2718 (2012)

17 Jin, S. W., Park, J., Hong, S. Y., Park, H., Jeong, Y. R., Park, J., Lee, S.-S. \& Ha, J. S. Stretchable loudspeaker using liquid metal microchannel. Sci. Rep. 5, 11695 (2015).

18 So, J.-H., Koo, H.-J., Dickey, M. D. \& Velev, O. D. Ionic current rectification in softmatter diodes with liquid-metal electrodes. Adv. Func. Mater. 22, 625-631 (2012).

19 Yoon, J., Hong, S. Y., Lim, Y., Lee, S.-J., Zi, G. \& Ha, J. S. Design and fabrication of novel stretchable device arrays on a deformable polymer substrate with embedded liquid-metal interconnections. Adv. Mater. 26, 6580-6586 (2014).

$20 \mathrm{Kim}$, H.-J., Son, C. \& Ziaie, B. A multiaxial stretchable interconnect using liquid-alloyfilled elastomeric microchannels. Appl. Phys. Lett. 92, 011904 (2008).

21 Kim, R.-H., Bae, M.-H., Kim, D. G., Cheng, H., Kim, B. H., Kim, D.-H., Li, M., Wu, J., Du, F., Kim, H.-S., Kim, S., Estrada, D., Hong, S. W., Huang, Y., Pop, E. \& Rogers, J. A. Stretchable, transparent graphene interconnects for arrays of microscale inorganic light emitting diodes on rubber substrates. Nano Lett. 11, 3881-3886 (2011).

22 Zheng, Y., He, Z.-Z., Yang, J. \& Liu, J. Personal electronics printing via tapping mode composite liquid metal ink delivery and adhesion mechanism. Sci. Rep. 4, 4588 (2014).

23 Gao, W., Emaminejad, S., Nyein, H. Y. Y., Challa, S., Chen, K., Peck, A., Fahad, H. M., Ota, H., Shiraki, H., Kiriya, D., Lien, D.-H., Brooks, G. A., Davis, R. W. \& Javey, A. Fully integrated wearable sensor arrays for multiplexed in situ perspiration analysis. Nature 529, 509-514 (2016).

24 Lee, H., Choi, T. K., Lee, Y. B., Cho, H. R., Ghaffari, R.-H., Wang, L., Choi, H. J., Chung, T. D., Lu, N., Hyeon, T., Choi, S. H. \& Kim, D.-H. A graphene-based electrochemical device with thermoresponsive microneedles for diabetes monitoring and therapy. Nat. Nanotechnol. 11, 566-572 (2016).

25 Kim, J., Banks, A., Cheng, H., Xie, Z., Xu, S., Jang, K.-I., Lee, J. W., Liu, Z., Gutruf, P., Huang, X., Wei, P., Liu, F., Li, K., Dalal, M., Ghaffari, R., Feng, X., Huang, Y., Gupta, S., Paik, U. \& Rogers, J. A. Epidermal electronics with advanced capabilities in near-field communication. Small 11, 906-912 (2015).

26 Kim, J., Salvatore, G. A., Araki, H., Chiarelli, A. M., Xie, Z., Banks, A., Sheng, X., Liu, Y., Lee, J. W., Jang, K.-I., Heo, S. Y., Cho, K., Luo, H., Zimmerman, B., Kim, J., Yan, L., Feng, X., Xu, S., Fabiani, M., Gratton, G., Huang, Y., Paik, U. \& Rogers, J. A. Battery-free, stretchable optoelectronic systems for wireless optical characterization of the skin. Sci. Adv. 2, 8 (2016).

27 Kim, B. H., Onses, M. S., Lim, J. B., Nam, S., Oh, N., Kim, H., Yu, K. J., Lee, J. W., Kim, J.-H., Kang, S.-K., Lee, C. H., Lee, J., Shin, J. H., Kim, N. H., Leal, C., Shim, M. \& Rogers, J. A. High-resolution patterns of quantum dots formed by electrohydrodynamic jet printing for light-emitting diodes. Nano Lett. 15, 969-973 (2015).

28 Graudejus, O., Görrn, P. \& Wagner, S. Controlling the morphology of gold films on poly (dimethylsiloxane). ACS Appl. Mater. Interfaces 2, 1927-1933 (2010).

29 Seghir, R. \& Arscott, S. Controlled mud-crack patterning and self-organized cracking of polydimethylsiloxane elastomer surfaces. Sci. Rep. 5, 14787 (2015).
31 Kramer, R. K., Boley, J. W., Stone, H. A., Weaver, J. C. \& Wood, R. J. Effect of microtextured surface topography on the wetting behavior of eutectic galliumindium alloys. Langmuir 30, 533-539 (2014).

32 Joshipura, I. D., Ayers, H. R., Majidi, C. \& Dickey, M. D. Methods to pattern liquid metals. J. Mater. Chem. C 3, 3834-3841 (2015).

33 Kramer, R. K., Majidi, C. \& Wood, R. J. Masked deposition of gallium-indium alloys for liquid-embedded elastomer conductors. Adv. Func. Mater. 23, 5292-5296 (2013)

34 Jeong, S. H., Hagman, A., Hjort, K., Jobs, M., Sundqvist, J. \& Wu, Z. Liquid alloy printing of microfluidic stretchable electronics. Lab Chip 12, 4657-4664 (2012).

$35 \mathrm{Li}$, G., Wu, X. \& Lee, D.-W. Selectively plated stretchable liquid metal wires for transparent electronics. Sens. Actuators, B 221, 1114-1119 (2015).

36 Kim, D., Thissen, P., Viner, G., Lee, D.-W., Choi, W., Chabal, Y. J. \& Lee, J.-B. Recovery of nonwetting characteristics by surface modification of gallium-based liquid metal droplets using hydrochloric acid vapor. ACS Appl. Mater. Interfaces 5, 179-185 (2013).

37 Kim, H. J., Maleki, T., Wei, P. \& Ziaie, B. A biaxial stretchable interconnect with liquidalloy-covered joints on elastomeric substrate. J. Microelectromech. Syst. 18, 138-146 (2009).

38 Cheng, S. \& Wu, Z. A microfluidic, reversibly stretchable, large-area wireless strain sensor. Adv. Func. Mater. 21, 2282-2290 (2011).

39 Park, Y.-L., Tepayotl-Ramirez, D., Wood, R. J. \& Majidi, C. Influence of cross-sectional geometry on the sensitivity and hysteresis of liquid-phase electronic pressure sensors. Appl. Phys. Lett. 101, 191904 (2012).

40 Kim, J., Banks, A., Xie, Z., Heo, S. Y., Gutruf, P., Lee, J. W., Xu, S., Jang, K.-I., Liu, F., Brown, G., Choi, J., Kim, J. H., Feng, X., Huang, Y., Paik, U. \& Rogers, J. A. Miniaturized flexible electronic systems with wireless power and near-field communication capabilities. Adv. Func. Mater. 25, 4761-4767 (2015).

41 Kaneko, A., Asai, N. \& Kanda, T. The influence of age on pressure perception of static and moving two-point discrimination in normal subjects. Hand Ther. 18, 421-425 (2005).

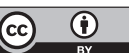

This work is licensed under a Creative Commons Attribution 4.0 International License. The images or other third party material in this article are included in the article's Creative Commons license, unless indicated otherwise in the credit line; if the material is not included under the Creative Commons license, users will need to obtain permission from the license holder to reproduce the material. To view a copy of this license, visit http:// creativecommons.org/licenses/by/4.0/

(C) The Author(s) 2017

Supplementary Information accompanies the paper on the NPG Asia Materials website (http://www.nature.com/am) 